\title{
Absolute sign of the Dzyaloshinskii-Moriya interaction in weak ferromagnets disclosed by polarized neutron diffraction
}

\author{
H. Thoma ${ }^{1,2}$, V. Hutanu ${ }^{1,2}$, G. Roth ${ }^{2}$, M. Angst ${ }^{3}$ \\ ${ }^{1}$ Jülich Centre for Neutron Science (JCNS) at Heinz Maier-Leibnitz Zentrum (MLZ), Forschungszentrum Jülich GmbH, 85748

 \\ Science JCNS and Peter Grünberg Institut PGI, JARA-FIT, Forschungszentrum Jülich GmbH, 52425 Jülich, Germany
}

\section{h.thoma@fz-juelich.de}

Magnetic interactions are the fundamental components for the fascinating variety of complex magnetic structures and properties found in many functional materials. Identifying, understanding, and finally predicting these interactions is an essential step towards their utilization in novel devices. One of these basic interactions is the Dzyaloshinskii-Moriya interaction (DMI) - an antisymmetric exchange coupling favouring a perpendicular arrangement of magnetic moments, and thus a canting in otherwise collinear structures $[1,2]$. The DMI, originally introduced in the late 1950s to explain 'weak ferromagnets' (not perfectly collinear antiferromagnets), regained the interest in current condensed matter research as it was found to be the driving force to stabilize various novel topological noncollinear magnetic structures, such as spin spirals [3], magnetic skyrmions [4], magnetic soliton lattices [5] and others. In particular for spintronic applications, the DMI shows promising characteristics towards the development of next-generation devices [6]. Although the magnitude of the DMI-induced canting is usually small, the direction can have a fundamental impact on the spin chirality and the resulting magnetic and multiferroic properties [7]. Here, we present polarized neutron diffraction (PND) as an efficient technique for the determination of the absolute direction of the DMI in weak ferromagnetic materials, as recently established by us [8].

We provide the basic formalism for a symmetry analysis of the DMI in crystal structures and show how to relate the measured PND data with the absolute DMI direction. We exemplify this approach in weak ferromagnetic $\mathrm{MnCO}_{3}$ and identify the magnetic moment configurations for a positive or negative sign of the DMI with an applied magnetic field as shown in Fig. 1. Using PND [9], we can distinguish even from the measurement of a single suitable Bragg reflection between the two configurations and unambiguously reveal a negative DMI sign in $\mathrm{MnCO}_{3}$. This is in agreement with previous results obtained by resonant magnetic X-ray scattering and thus, validates the method [10]. We demonstrate the generality of our method by providing further examples of topical magnetic materials with different symmetries and support our findings with ab-initio calculations, which reproduce the experimental results.
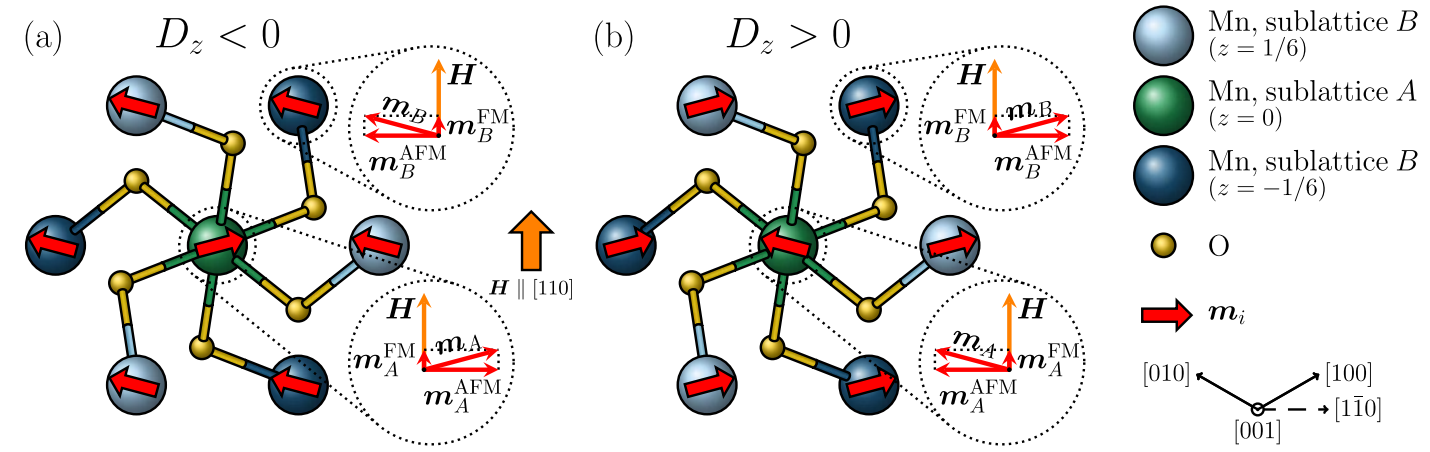

Figure 1. The local environment of the $\mathrm{z}=0$ manganese atom in the hexagonal unit cell of $\mathrm{MnCO}_{3}$. The six nearest-neighbour manganese atoms of the other magnetic sublattice are shown as light and dark blue spheres located above and below the central atom, respectively. The oxygen atoms between these manganese layers are shown as small yellow spheres. Panels (a) and (b) show the two possible magnetic moment configurations stabilized dependent on the sign of the $D_{z}$ DMI component by applying an external magnetic field along the [110] direction aligning the weak ferromagnetic moment.

[1] V. E. Dzyaloshinskii, Sov. Phys. - JETP 5(6), 1259 (1957)

[2] T. Moriya, Phys. Rev. 120(1), 91 (1960)

[3] M. Bode et al., Nature 447, 190 (2007)

[4] S. Heinze et al., Nat. Phys. 7, 713 (2011)

[5] Y. Togawa et al., Phys. Rev. Lett. 108, 107202 (2012)
[6] S. S. P. Parkin et al., Science 320, 190 (2008)

[7] J. Cho et al., J. Phys. D: Appl. Phys. 50, 425004 (2017)

[8] H. Thoma et al., Phys. Rev. X 11, 011060 (2021)

[9] H. Thoma et al., J. Appl. Crystallogr. 51, 17 (2018)

[10] V. E. Dmitrienko et al., Nat. Phys. 10, 202 (2014)

Keywords: Dzyaloshinskii-Moriya interaction; weak ferromagnets; polarized neutron diffraction; magnetic structures 